\title{
Intraoperative Tracheostomy Tube Customization
}

\author{
Anne Hseu ${ }^{1}$, Zain Ul Abedeen Sobani ${ }^{2}$, Joseph Scharpf ${ }^{1^{*}}$ \\ ${ }^{1}$ Head \& Neck Institute, Cleveland Clinic Foundation, Cleveland, USA \\ ${ }^{2}$ Aga Khan University, Karachi, Pakistan \\ Email: ${ }^{*}$ scharpj@ccf.org
}

Received April 7, 2013; revised May 10, 2013; accepted June 15, 2013

Copyright (C) 2013 Anne Hseu et al. This is an open access article distributed under the Creative Commons Attribution License, which permits unrestricted use, distribution, and reproduction in any medium, provided the original work is properly cited.

\begin{abstract}
Objectives: This article describes how to make a customized tracheostomy tube immediately in the operating room setting. This is particularly critical when a commercial customized tracheostomy tube cannot be readily obtained. Study Design: Case presentation. Methods/Results: A 73-year-old female was seen in our clinic for management of a recurrent invasive paraganglioma of the thyroid. She underwent a total laryngopharyngectomy, cervical esophagectomy, and anterolateral thigh free flap reconstruction followed by post-operative radiation. In follow-up, the patient presented with dyspnea related to two areas of stenosis, one at the level of her stoma and one at the distal trachea. The patient was therefore taken to the operating room urgently for dilation and placement of a tracheostomy tube. Available tracheostomy tubes were tried and ill fitting as each tube narrowed the patient's stoma or abutted her distal granulation tissue. To custom create a tracheostomy tube, we used a standard rib shearer to shorten a \#6 uncuffed tracheostomy tube by 2 $\mathrm{cm}$. The edges were further smoothed and beveled using sand paper and a diamond burr drill. The finished product was a wide diameter tube with a custom length suited to our patient. Conclusions: Although a simple solution, the use of a rib shearer provides a quick and feasible solution to creating custom length tracheostomy tubes in situations where custom length tubes are needed yet unavailable.
\end{abstract}

Keywords: Tracheostomy Tube; Customized

\section{Introduction}

Tracheotomies are amongst the most common neck procedures performed and essential for long-term airway management. Although often straight forward, the procedure can be made technically difficult in short statured, short necked, or obese patients [1]. In such patients, unfavorable anatomy can include exaggerated curvature of the spine, elongated depth from skin to trachea, lateral curvature of the trachea, or a short cricoid to sternal distance $[2,3]$. These anatomical differences often make standard tracheostomy tubes unsuitable. Subsequently, illfitting tubes are more prone to dislodgement and are associated with increased morbidity and mortality [4].

Although custom tracheostomy tubes can often be ordered and express mailed, we have encountered urgent patient scenarios where custom tubes are needed but not available. Here we present a simple yet effective technique to modify standard cuffless tracheostomy tubes immediately in the operating room setting.

\footnotetext{
${ }^{*}$ Corresponding author.
}

\section{Case Presentation}

A 73-year-old female presented to our clinic for management of a recurrent invasive paraganglioma of the right thyroid. She had previously undergone two prior resections at outside institutions. At presentation, the patient was noted to have recurrence with gross invasion into her esophagus and trachea. She therefore underwent a total laryngopharyngectomy, cervical esophagectomy, and anterolateral thigh (ALT) free flap reconstruction followed by post-operative radiation. The patient's course was complicated by a significant degree of stomal stenosis. Her stoma was initially dilated in the office to a \#6 diameter tracheostomy tube; any smaller diameter tube did not provide her a sufficient airway.

Stomal revision was deferred given the patient's overall poor medical condition.

Unfortunately, in follow-up, the patient presented with worsening dyspnea and tube discomfort. Flexible endoscopic examination revealed that granulation tissue had developed at the left inferior aspect of the distal tracheostomy tube. The patient was therefore taken to the oper- 
ating room and further assessment of the airway revealed that the ideal tracheostomy tube would be a shorter length \#6 tube. A smaller diameter tube would not afford the patient a sufficient airway at the level of the stoma and a standard \#6 tube would continue to irritate the area of tracheal wall granulation.

\section{Methods/Results}

In order to custom create a tracheostomy tube for our patient, we used a standard rib shearer (Figure 1) to shorten a \#6 cuffless Air-Lon tracheostomy tube by approximately $2 \mathrm{~cm}$. After shortening the tube's inner and outer cannulas, we further smoothed and beveled the edges of the tubes using sand paper and a diamond burr drill. The finished product was a wide diameter tube with a custom length suited to our patient. This temporary, customized tracheostomy tube took minimal steps to make and could be fashioned with any non-metal cuffless tracheostomy tube.

Once the custom-length tracheostomy tube was placed, we re-examined our patient with a flexible endoscope. The tube sat in an excellent position well above the previously noted granulation tissue and afforded the patient an appropriate diameter at the level of stomal stenosis.

\section{Discussion}

In the literature, there are several descriptions to create custom tracheostomy tubes [5-7]. Ideally, appropriate length tracheostomy tubes would always be available for patients. There are circumstances, however, when this is not the case despite a large array of tracheostomy tubes available at our institution.

In our case, our patient unexpectedly presented with dyspnea related to two areas of stenosis-one at the level of her stoma and one related to granulation tissue distally. Given this, it was not plausible to decannulate the patient for an extended period of time and her worsening symptoms dictated that we act urgently. In the operating room, a number of tracheostomy tubes were attempted but not ideal. Each tube either abutted the granulation tissue or narrowed the stoma too much to allow for comfortable ventilation. Short of placing an endotracheal tube and keeping the patient on a ventilator, good treatment options without a custom length tube were limited.

This case demonstrates a process to create custom length tracheostomy tubes in any operating room using readily available tools. Albeit a simple solution, the use of a rib shearer is quick and feasible with the entire process taking a matter of minutes. Our method of customizing tracheotomy tubes is an easy process that can be applied to situations where custom length tracheostomy tubes are needed yet unavailable. We hope that this straightforward method allows patients and surgeons

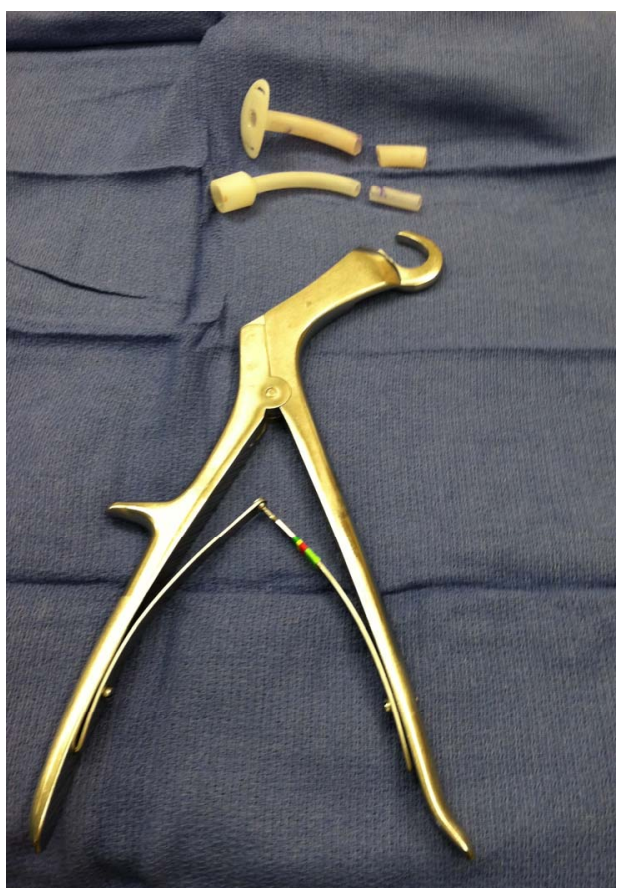

Figure 1. Display of rib shearer and tracheostomy tube outer and inner cannulas after custom shortening.

to safely traverse to the point when a long-term custom manufactured tracheostomy tube can be ordered and placed.

\section{Conclusion}

Although a simple solution, the use of a rib shearer provides a quick and feasible solution to creating custom length tracheostomy tubes in situations where custom length tubes are needed yet unavailable.

\section{REFERENCES}

[1] S. M. Hwang, J. S. Jang, J. I. Yoo, et al., "Difficult Tracheostomy Tube Placement in an Obese Patient with a Short Neck-A Case Report," Korean Journal of Anesthesiology, Vol. 60, No. 6, 2011, pp. 434-436. doi:10.4097/kjae.2011.60.6.434

[2] J. K. Muhammad, E. Major and D.W. Patton, "Evaluating the Neck for Percutaneous Dilatational Tracheostomy," Journal of Cranio-Maxillofacial Surgery, Vol. 28, No. 6, 2000, pp. 336-342. doi:10.1054/jcms.2000.0170

[3] A. Tabaee, E. Geng, J. Lin, et al., "Impact of Neck Length on the Safety of Percutaneous and Surgical Tracheotomy: A Prospective, Randomized Study,” Laryngoscope, Vol. 115, No. 9, 2005, pp. 1685-1690. doi:10.1097/01.MLG.0000175539.25182.2A

[4] N. D. Gross, J. I. Cohen, P. E. Andersen and M. K. Wax, "“Defatting' Tracheotomy in Morbidly Obese Patients," Laryngoscope, Vol. 112, No. 11, 2002, pp. 1940-1944. doi:10.1097/00005537-200211000-00006

[5] T. Ma and J. C. Ross, “A Technique for Fabrication of a 
Custom Tracheostomy Prosthesis," Journal of Prosthetic Dentistry, Vol. 62, No. 6, 1989, pp. 668-671.

doi:10.1016/0022-3913(89)90588-X

[6] R. F. Busch, "An Immediate Custom Tracheostomy Tube for the Thick Neck,” Otolaryngology_Head and Neck
Surgery, Vol. 109, No. 5, 1993, pp. 959-961.

[7] B. K. Reilly and M. S. Volk, "Emergency Use of a Customized Tracheostomy Tube for Pediatric Patients," Laryngoscope, Vol. 122, No. 7, 2012, pp. 1624-1625. doi:10.1002/lary.23267 